\title{
Ultra-fast relaxation rates and reversal time in disordered ferrimagnets
}

\author{
O.J. Suarez ${ }^{1,2}$, P. Nieves ${ }^{3}$, D. Laroze ${ }^{1,4}$, D. Altbir ${ }^{5}$, and O. Chubykalo-Fesenko ${ }^{3}$ \\ ${ }^{1}$ Instituto de Alta Investigación, Universidad de Tarapacá, Casilla 7D, Arica, Chile \\ ${ }^{2}$ Departamento de Matemáticas y Física, Universidad de Sucre, A.A. 406, Sincelejo, Colombia \\ ${ }^{3}$ Instituto de Ciencia de Materiales de Madrid, CSIC, Cantoblanco, 28049 Madrid, Spain \\ ${ }^{4}$ SUPA School of Physics and Astronomy, University of Glasgow, Glasgow G128QQ, UK and \\ ${ }^{5}$ Departamento de Física, CEDENNA, Universidad de Santiago de Chile, Av. Ecuador 3493, Santiago, Chile
}

(Dated: October 2, 2015)

\begin{abstract}
In response to the ultra-fast laser pulses single-phase metals have been classified as "fast" (with magnetization quenching on the timescale below $100 \mathrm{fs}$ and recovery in the timescale of several ps and below) and "slow" (with longer characteristic time scales). The disordered ferrimagnetic alloys consisted of a combination of "fast" transition (TM) and "slow" rare earth (RE) metals have been shown to exhibit the ultra-fast all-optical switching mediated by the heat mechanism. The behavior of characteristic timescales of coupled alloys is more complicated and is influenced by many parameters such as the inter-sublattice exchange, doping (RE) concentration and the temperature. Here the longitudinal relaxation times of each sublattice are analyzed within the Landau-Lifshitz-Bloch framework. We show that for moderate inter-sublattice coupling strength both materials slow down as a function of "slow" (RE) material concentration. For larger coupling the "fast" (TM) material may fasten while the "slow" (RE) one is still slower. These conclusions may have important implications in the switching time of disordered ferrimagnets such as GdFeCo with partial clustering. Using the atomistic modeling we show that in the moderately coupled case the reversal would start in Gd-rich region, while the situation may be reversed if the coupling strength is larger.
\end{abstract}

PACS numbers: 75.78.Jp, 75.40.Mg, 75.40.Gb

\section{INTRODUCTION}

The possibility to switch magnetization at the ultra-fast timescale with laser pulses has been fascinating the scientific community for already more than 15 years. ${ }^{1}$ This discovery could potentially have important technological implications since it promises much faster magnetic recording without the need of any recording head. ${ }^{2}$ The future applications also require an accurate understanding and design of the corresponding materials. Until now, the ultra-fast heat-mediated switching with linearly polarised laser pulses has been observed only in the antiferromagnetically coupled materials such as disordered ferrimagnets $\mathrm{GdFeCo},{ }^{3} \mathrm{TbCo}^{4}$ and $\mathrm{TbFe} .{ }^{5}$ In addition, by using the circularly-polarized lasers, another switching mechanism, based on the inverse Faraday effect, may be also feasible. ${ }^{7}$ Recently, the all-optical switching by circularpolarised lasers has been also reported in other multilayers and heterostructures, including the ferromagnetic ones. ${ }^{6} \mathrm{How}-$ ever, the pure thermal switching mechanism ${ }^{8}$ under linearly polarised laser pulses seems to occur in antiferromagneticallycoupled materials only.

These materials consist of sublattices with different responses to ultra-fast laser excitation in terms of the demagnetization speeds: fast transition metal (TM) slow rare earth (RE) material. The notion of "fast" and "slow" materials has been introduced in Ref. ${ }^{9}$ and is based on the classification of materials according to their responses to the laser pulse: the TM typically demagnetize in the timescale of $100 \mathrm{fs}$ and recover the magnetisation in several ps timescale while the RE materials have two-stage demagnetisation process and recover the magnetization in the timescale above 100ps. The classification is based on the parameter $\lambda J / \mu$, describing the longitudinal relaxation rate, ${ }^{10}$ where $J$ is the exchange parameter (proportional to the Curie temperature $T_{C}$ ), $\mu$ is the magnetic moment and $\lambda$ is the coupling to the bath parameter (proportional to the spin-flip probability and coinciding with the atomic damping parameter). This parameter is clearly smaller for rare earth materials than for transition metals and thus, the first are classiffied as "slow" materials (with picosecond timescale longitudinal relaxation) and the second - as "fast" materials (with femtosecond timescale relaxation).

The magnetization dynamics in ferrimanetic materials is more complicated due to the presence of two sublattices with intrinsically non-equivalent "speeds" which are exchangely coupled. The switching involves the angular momentum transfer from the ferromagnetic precessional mode to the antiferromagnetic one. ${ }^{11}$ Under the influence of the laser pulse the fast material arrives at almost zero magnetization. At this moment the magnetization of the slow material has a nonzero value and provides an exchange field acting on the fast material. The switching is fast due to the fact that it occurs in a strong exchange field. Therefore, one could expect that the most efficient switching would correspond to the situation where the difference in the speed in two materials is the highest. The XMCD studies ${ }^{3}$ confirmed the different demagnetization speeds in each material of the alloy. Recent experiment ${ }^{12}$ using novel synchrotron radiation techniques have shown that in $\mathrm{GdFeCo}$ a partial clustering of $\mathrm{Gd}$ occurs and the switching starts in Gd-rich regions.

In this article we analyze the demagnetization speed and the switching time of the two materials constituting a generic ferrimagnetic alloy as a function of their concentration. The main features of the ultra-fast dynamics in these materials are very well described by the atomistic simulations based on the Heisenberg model with Langevin dynamics. ${ }^{3,11}$ However, the Landau-Lifshitz-Bloch (LLB) approach, ${ }^{13}$ based on the thermal averaged dynamics, is very convenient for analyt- 
ical treatment and provides a general picture of the problem.

This paper is organized as follows. In Sec. II we describe our theoretical model, whereas in Sec. III we present the results and discussion. Finally, the conclusions are summarized in Sec. IV.

\section{THEORETICAL MODEL}

The recently derived Landau-Lifshitz-Bloch equation $(\mathrm{LLB})^{13}$ for classical ferrimagnets valid up to the Curie temperature, it is a suitable tool to rapidly understand the difference in demagnetization speeds of two-component coupled materials within an analytical framework. Neglecting the transverse components, i.e., assuming that the magnetization of each sublattice is parallel to the effective field, this equation is given by ${ }^{13}$

$$
\frac{d m_{v}}{d \tau}=\left[\frac{1}{2 \Lambda_{v \kappa}}\left(\frac{m_{\kappa}^{2}}{m_{e, \mathrm{\kappa}}^{2}}-1\right)-\frac{1}{2 \Lambda_{v v}}\left(\frac{m_{v}^{2}}{m_{e, v}^{2}}-1\right)\right] m_{v},
$$

where $\tau=\gamma_{v} \alpha_{\|}^{v} t$ is the reduced time, $m_{v}$ and $m_{\mathrm{K}}$ are the sublattice magnetizations, $\gamma_{v}$ is the gyromagnetic ratio, $\alpha_{\|}^{v}=$ $\left(2 \lambda_{v} m_{e, v}\right) /\left(\beta \mu_{v} H_{v, e}^{e x}\right)$ is longitudinal damping parameter, $\lambda_{v}$ is the (atomistic) coupling to the bath parameter, $\beta=1 / k_{B} T$, $k_{B}$ is the Boltzmann constant, $T$ is the temperature, $\mu_{v}$ is the magnetic moment and the sub-index $e$ denotes the equilibrium values. The exchange field acting on the sublatice $v$ in the mean-field approximation (MFA) has the following form:

$$
\mu_{v} H_{v}^{e x}=J_{0, v} m_{v}+\left|J_{0, v \kappa}\right| m_{\kappa}
$$

with $J_{0, v}=x_{v} z J_{v v}, J_{0, v \kappa}=x_{\kappa} z J_{v \kappa}, z$ is the number of nearest neighbours in the ordered lattice, $x_{\mathrm{v}}$ is the concentration of the material $v\left(x_{v}+x_{\mathrm{K}}=1\right), J_{v v}, J_{v K}$, are the Heisenberg intra and inter-sublattice exchange interaction parameters. The quantities $\Lambda_{v v}$ and $\Lambda_{v \kappa}$ are given by ${ }^{13,15}$

$$
\Lambda_{\mathrm{vv}}^{-1}=\frac{1}{\widetilde{\chi}_{v, \|}}\left(1+\frac{J_{0, v \kappa}}{\mu_{v}} \widetilde{\chi}_{\kappa, \|}\right), \quad \Lambda_{\mathrm{v \kappa}}^{-1}=\frac{m_{e, \mathrm{\kappa}}}{m_{e, v}} \frac{\left|J_{0, v \kappa}\right|}{\mu_{v}},
$$

where $\widetilde{\chi}_{v,||}$ is the longitudinal susceptibility which in the MFA is given by

$$
\widetilde{\chi}_{v, \|}=\frac{\mu_{\kappa} \beta L_{v}^{\prime} J_{0, v \kappa} \beta L_{\kappa}^{\prime}+\mu_{v} \beta L_{v}^{\prime}\left(1-J_{0, \kappa} \beta L_{\kappa}^{\prime}\right)}{\left(1-J_{0, v} \beta L_{v}^{\prime}\right)\left(1-J_{0, \kappa} \beta L_{\kappa}^{\prime}\right)-J_{0, \kappa v} \beta L_{v}^{\prime} J_{0, v \kappa} \beta L_{\kappa}^{\prime}},
$$

where $L_{v}^{\prime}(\xi)=d L_{v} / d \xi$ is the derivative of the Langevin function $L(\xi)=\operatorname{coth}(\xi)-1 / \xi$ evaluated at $\xi=\xi_{e, v}=\beta \mu_{v} H_{v, e}^{e x}$. The quantity $m_{e, v}$ can be evaluated in the MFA solving the system of the Curie-Weiss equations: $m_{v}=L\left(\xi_{v}\right)$.

Recently, ${ }^{14}$ the ferrimagnetic LLB equation has been also extended for temperatures above the Curie temperature where it coincides with the linearized form of Eq. (1). Namely, close and above to $T_{C}$ the longitudinal and the transverse damping parameters coincide and are equal to

$$
\alpha_{\|}^{v} \simeq \frac{2 \lambda_{v} T}{3 T_{C}}
$$

Additionally, the longitudinal susceptibility given by Eq. (4) becomes

$$
\tilde{\chi}_{v, \|}=\frac{\mu_{\kappa} J_{0, v \kappa} \beta^{2}+\mu_{v} \beta\left(3-J_{0, \kappa} \beta\right)}{\left(3-J_{0, v} \beta\right)\left(3-J_{0, \kappa} \beta\right)-J_{0, \kappa v} J_{0, v \kappa} \beta^{2}}, \quad T>T_{C} .
$$

Particularly, it is been shown that the presence of two nonequivalent sublattices removes the true critical slowing down at the Curie temperature since all the parameters in Eq. (1) are finite. This could be understood as following from the fact that the sublattices dynamics occurs in the field coming from the other sublattice.

In what follows we will call the "fast" material - transition metal (denoted by $T M$ ) and the slow material- rare earth (denoted by $R E$ ). The "fast" character of TM will be defined by larger exchange parameter and smaller magnetic moment as the usual case in the TM-RE alloys. Furthermore, for simplicity in notation, we denote the intra and inter-sublattice coupling parameters as: $J_{T M-T M}=J_{T T}, J_{R E-R E}=J_{R R}$ and $J_{T M-R E}=J_{T R}$. The concentration of the RE and TM materials are $x_{R E}$ and $x_{T M}$, respectively.

To calculate the relaxation rates of each sublattice, the system of coupled LLB equations (1) is linearized. This gives a characteristic matrix, $\mathcal{A}_{\|}$, which drives the dynamics of the linearized equation $\partial(\delta \mathbf{m}) / \partial t=\mathcal{A}_{\|} \delta \mathbf{m}$. The matrix $\mathcal{A}_{\|}$reads

$$
\mathcal{A}_{\|}=\left(\begin{array}{cc}
-\gamma_{\mathrm{TM}} \alpha_{\|}^{\mathrm{TM}} / \Lambda_{\mathrm{TT}} & \gamma_{\mathrm{TM}} \alpha_{\|}^{\mathrm{TM}} J_{0, \mathrm{TR}} / \mu_{\mathrm{T}} \\
\gamma_{\mathrm{RE}} \alpha_{\|}^{\mathrm{RE}} J_{0, \mathrm{RT}} / \mu_{\mathrm{R}} & -\gamma_{\mathrm{RE}} \alpha_{\|}^{\mathrm{RE}} / \Lambda_{\mathrm{RR}}
\end{array}\right)=\left(\begin{array}{cc}
-\Gamma_{\mathrm{TT}} & \Gamma_{\mathrm{TR}} \\
\Gamma_{\mathrm{RT}} & -\Gamma_{\mathrm{RR}}
\end{array}\right) .
$$

It is important to note that the matrix elements in Eq. (7) are temperature and material parameter dependent. The general solution of the characteristic equation, $\left|\mathcal{A}_{\|}-\Gamma^{ \pm} I\right|=0$, gives two different eigenvalues

$$
\Gamma^{ \pm}=\frac{-\left(\Gamma_{\mathrm{TT}}+\Gamma_{\mathrm{RR}}\right) \pm \sqrt{\left(\Gamma_{\mathrm{TT}}-\Gamma_{\mathrm{RR}}\right)^{2}+4 \Gamma_{\mathrm{RT}} \Gamma_{\mathrm{TR}}} .}{2} .
$$

In ferromagnets, relaxation can usually be described well by only one relaxation rate, at least in the linear regime. By contrast, in two sublattice ferrimagnets, in the most general case one cannot describe the relaxation with a single exponential decay.

To be more specific, we consider a GdFeCo alloy material with the sublattices $\{\mathrm{RE}, \mathrm{TM}\}=\{\mathrm{Gd}, \mathrm{FeCo}\}$. We assume a model of $G d_{x_{R E}}(\mathrm{FeCo})_{x_{T M}}$ alloy with typical atomistic Heisenberg parameters from Ref.: ${ }^{11} J_{\text {TT }}^{\text {atom }}=6.92 \cdot 10^{-21}$ $\mathrm{J}, J_{\mathrm{RR}}^{\text {atom }}=2.78 \cdot 10^{-21} \mathrm{~J}$ and $J_{\mathrm{TR}}^{\text {atom }}=-2.41 \cdot 10^{-21} \mathrm{~J}$. The magnetic moments used are $\mu_{T M}=1.92 \mu_{B}$ and $\mu_{R E}=7.63 \mu_{B}$ where $\mu_{B}$ is the Bohr magneton. Furthermore, the relaxation rates are just proportional to the atomistic coupling to the bath parameter. This parameter is normally extracted from the experiment. For simplicity we have fixed it for each sublattice to the value, $\lambda_{T M}=\lambda_{R E}=0.1$ used previously. ${ }^{13}$ Since the LLB equation uses the MFA, the exchange parameters were renormalized using the standard procedure ${ }^{16}$ to obtain the same Curie temperature as in the atomistic simulations and experiments. Furthermore, the generic character of the ferrimagnet is simulated by varying the antiferromagnetic coupling strength, $J_{T R}$. In what follows we will denote the typical value of the atomistic exchange strength for $\mathrm{GdFeCo}$ as 


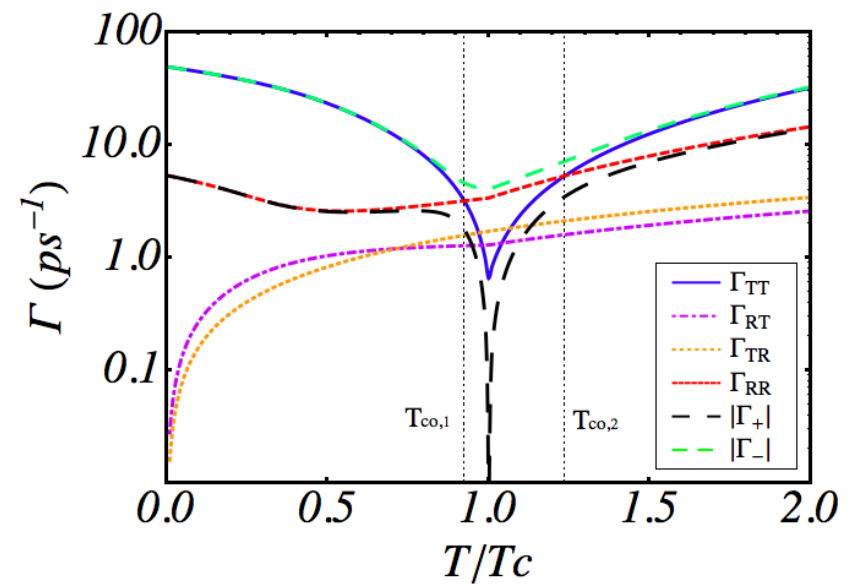

FIG. 1. (Color online) The matrix elements of $\mathcal{A}_{\|}$and $\Gamma^{ \pm}$as a function of the temperature using a rare-earth concentration of $x_{R E}=0.25$ for typical $\mathrm{GdFeCo}$ parameters.

$J_{1}^{\text {atom }} \equiv J_{\mathrm{TR}}^{\text {atom }}$ and its normalized for MFA value as $J_{1}$. Note that the two-sublattices have a shared Curie temperature except for a very small inter-sublattice coupling.

\section{RESULTS AND DISCUSSION}

In this section we present our results of the relaxation rates for ferrimagnetic alloys obtained within the framework of the Landau-Lifshitz- Bloch model. We begin showing in Fig. 1 the matrix elements of $\mathcal{A}_{\|}$and the combined rates $\Gamma^{ \pm}$as a function of the reduced temperature $T / T_{C}$ using the concentration $x_{R E}=0.25$. The temperature where $\Gamma_{T T}=\Gamma_{R R}$ is called the coupling temperature $T_{\mathrm{co}} \cdot{ }^{15}$ For relatively low temperatures, $T / T_{C}<T_{\mathrm{co}, 1}$ and for temperatures $T / T_{C}>T_{\mathrm{co}, 2}$, we have $\Gamma_{T R}, \Gamma_{R T} \ll \Gamma_{T T}, \Gamma_{R R}$. Therefore, the longitudinal relaxation time of each sublattice in these temperature regions can be written as $\tau_{v}^{\|} \simeq 1 / \Gamma_{v v}$. Thus each subsystem could be characterized by the corresponding longitudinal time $\tau_{T M, R E}^{\|}$, although we should stress that the rates $\Gamma_{R R}, \Gamma_{T T}$ depend on parameters of both subsystems. Taking into account that the exchange field is large, one can estimate the longitudinal relaxation time of each sublattice in this region as

$$
\tau_{v}^{\|} \approx \frac{1}{2 \gamma_{v} \lambda_{v} m_{v, e} H_{v, e}^{e x}}
$$

In the region $T_{\mathrm{co}, 1}<T / T_{C}<T_{\mathrm{co}, 2}$, i.e. near $T_{C}$, the linearized relaxation dynamics of each subsystem should be characterized by two characteristic exponents $\Gamma^{ \pm}$. We called this regime "coupled" dynamics. One can also obtain from Eq. (4) that close to $T_{C}$ the susceptibilities diverge, namely $\chi_{v, \|} \propto 1 /\left|T-T_{C}\right|$ which is the main source for the critical slowing down of the longitudinal relaxation times for singlespecie materials near the Curie temperature. As we mentioned above, the true critical slowing down (infinite relaxation time) does not occur for two-component materials. However, the relaxation time still show an increment at the Curie temperatures.

The temperature dependence of $\tau_{T M, R E}^{\|}$(obtained from the evaluation of $1 / \Gamma_{T T, R R}$ given by Eq. (7)) varying the intersublattice exchange strength are presented in Fig. 2 ( $a$ and b) for $x_{R E}=0.25$, and in Fig. 2 (c and d), varying the RE concentration and fixing the inter-sublattice exchange. For large temperatures, the TM material experiences critical slowing down although, differently to pure ferromagnetic materials, the relaxation time remains finite. The behavior of the RE material is more complicated. Namely, only for very large coupling the critical slowing down at common Curie temperature occurs. In all other cases, one more peak appears, corresponding to the slowing down at temperatures roughly equal to the individual Curie temperature of the RE material.

The functional dependence of relaxation times can be understood taken into account that it is inversely proportional to the exchange field through Eq. (9), which in turn is proportional to the inter-sublattice exchange field. As the coupling strength increases, the MFA exchange field increases, and thus, both RE and TM become slower. At the same time, the dependence of the exchange field on the RE concentration via Eq. (2) is more complicated. Effectively, on the MFA level, small RE concentration means large coupling for the RE since the effective field from the TM sublattice is large in this case. On the contrary, small RE concentration means small coupling for the TM. It follows from Eqs. (9) and (2) that for $J_{T M} m_{T M}>\left|J_{T R}\right| m_{R E}$ the exchange field acting on the TM decreases with the rare earth concentration and the TM material becomes slower in agreement with Fig. 2c). The same happens for the RE elements if $\left|J_{T R}\right| m_{T M}>J_{R E} m_{R E}$, in agreement with Fig. 2d). At the same time it is clear that for larger inter-sublattice exchange coupling the above inequality for the TM metal can be violated, while for the RE it always holds. Thus, we can have situation in which TM fastens with concentration while the RE still slows down. The transition between the two behaviors is depicted in Fig. 3, where we present only concentration values typical for the experiment and corresponding to the window where the magnetization switching could take place. The decrease of the TM relaxation time with concentration for large inter-sublattice exchange strength indicates that one can expect a non-trivial behavior as a function of the concentration.

At temperatures close to the Curie temperature, i.e. $T_{c o, 1}<$ $T / T_{C}<T_{c o, 2}$ the estimation of the relaxation rates based on only one eigenvalue is not valid. Besides, the system dynamics becomes highly nonlinear. However, frequently the magnetization quenching at ultra-fast timescale is characterized experimentally by one characteristic time $\tau_{M}$. To comply with this procedure, we fit the numerically integrated relaxation time at high temperatures to one exponential function for each sub-lattice. The integration of the original LLB equation (1) is performed with initial conditions taken as a small deviations of each sublattice from its equilibrium, i.e., $m_{\mathrm{v}}(0)=m_{e, v}+\delta m_{e, v}$. The results for three temperatures in the coupling regime, in comparison with the analytically estimated relaxation time (based on Eq. (9)) are presented in Fig. 4. One observes that, firstly, the linear one-exponential 

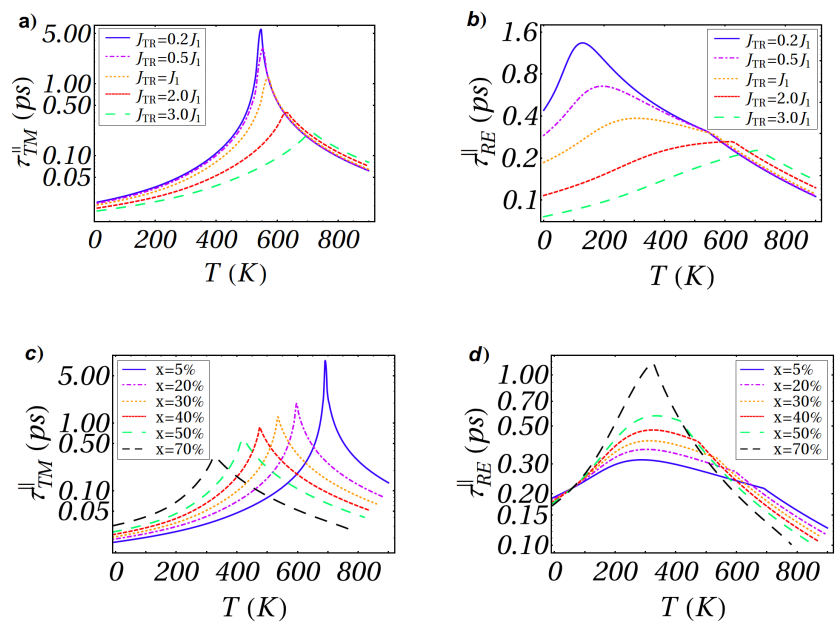

FIG. 2. (Color online) Temperature dependence of the longitudinal relaxation times $\tau_{T M, R E}$ for different values of a), b) Inter-sublattice coupling strength $J_{T R}$ and c), d) different concentrations and $J_{T R}=$ $J_{1}$.
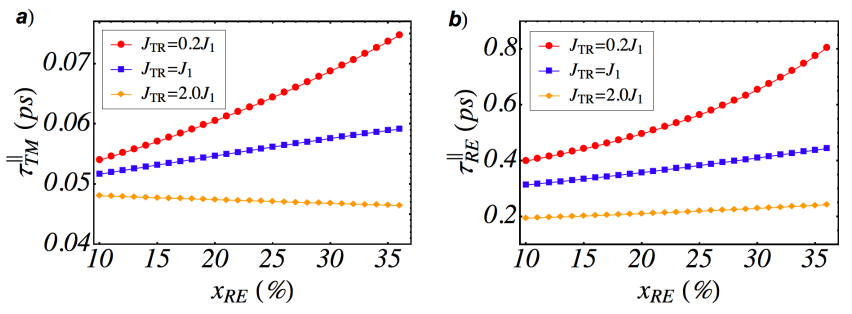

FIG. 3. (Color online) The relaxation times a) $\tau_{\mathrm{TM}}^{\|}$and b) $\tau_{\mathrm{RE}}^{\|}$obtained from the evaluation of $1 / \Gamma_{T T}$ and $1 / \Gamma_{R R}$, respectively, as a function of the rare-earth concentration for different strength couplings between both sublattices at $T=0.6 T_{C}$.

approximation is still valid for small coupling strength. Secondly, at high temperatures the RE accelerates as a function of temperature while the TM slows down. This phenomena is also present in the behavior of the matrix elements and the combined rates in Fig. 1 and in Fig. 2 and can be explained by the fact that while the TM has a critical behavior at the common Curie temperature, the RE material presents a strong critical behavior in the temperature, close to its own Curie temperature. At temperatures close to $T_{C}$ its relaxation time decreases. We should stress again that Fig. $4 \mathrm{c}$ ) is calculated for the parameters for which the system is in the strongly coupled regime and cannot be characterized by one relaxational time. In this case the LLB and analytical approach give different results as a function of concentration, namely close to $T_{C}$ the analytical result predicts the decrease of the relaxation time with concentration while the direct LLB integration shows a slight increase.

The analysis of the longitudinal components indicates the regions of the parameters with different material's response which is a necessary condition for switching. However, this is not a sufficient condition. In fact, Eq. (1) does not describe
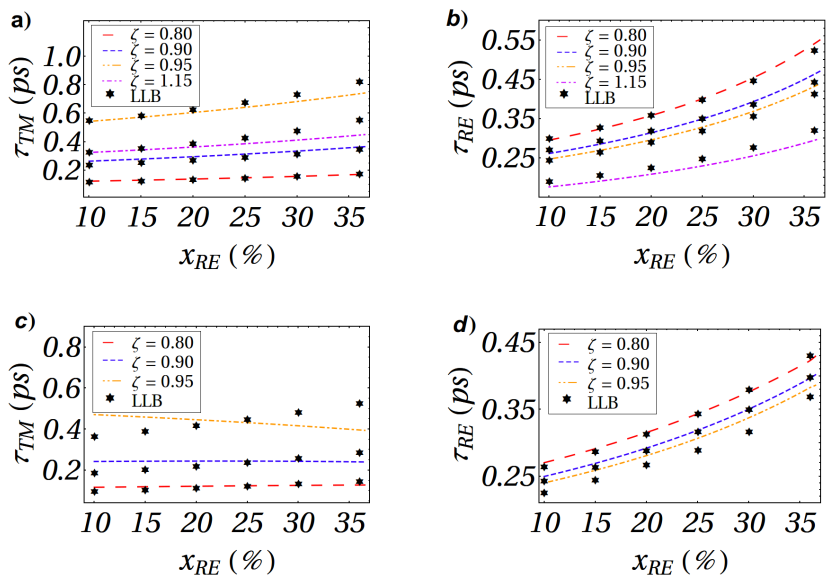

FIG. 4. (Color online) The relaxation times $\tau_{T M}$ and $\tau_{R E}$ as a function of the rare-earth concentration, for different reduced temperatures, $\zeta=T / T_{C}$. The lines indicate the relaxation times obtained via the evaluation of the eigenvalues Eq. (7) while the points indicate the direct numerical integration of the LLB Equation and fit to the oneexponential function. The Figs. a), b) correspond to the coupling strength values $J_{T R}=0.2 J_{1}$, and Figs. c), d) for $J_{T R}=J_{1}$.

magnetization switching since the lines with zero magnetisation cannot be crossed by a pure longitudinal motion. For a proper switching, the longitudinal motion should transfer angular momentum to the transverse motion. ${ }^{17}$ The best approach to simulate the switching is the atomistic one which we use below. However, the above results can have consequences on the switching because the angular momentum transfer is efficient when at the moment when TM arrives at zero magnetisation, the RE has large magnetisation value.

To analyse the consequences of the predicted relaxation times on the switching of a ferrimagnet, we can first adopt a usually made simplification by considering a step function for the electronic temperature (see, for example Refs. ${ }^{11,15}$ ) We also know from atomistic simulations that the switching occurs well after the laser pulse is gone. Thus although the electronic temperature may go above the Curie temperature, the relaxation time of a sublattice during the ultra-fast laserinduced switching may be approximately characterized by its relaxation at the final (quasi-equilibrium) electron temperature which is below $T_{C}$. The switching in GdFeCo will be effective if $\tau_{T M}$ is small and $\tau_{R E}$ is large, since in this case we could expect a large field acting on the TM material. This reasoning indicates that if the quasi-equilibrium temperature stays close to $T_{C}$, the switching is ineffective, since in this case the TM relaxation time is as large as that of the RE. Thus the switching should be suppressed for large pulse intensities. The switching diagram as a function of the temperatures can be found in Ref., 11,15 and indicates that the switching is indeed suppressed for high temperatures. Here we are interested to understand the phenomena as a function of the RE concentration.

To analyse the switching, we perform atomistic modeling of a disordered GdFeCo ferrimagnet under the influence of ultra-fast laser pulse. The model is described in details 


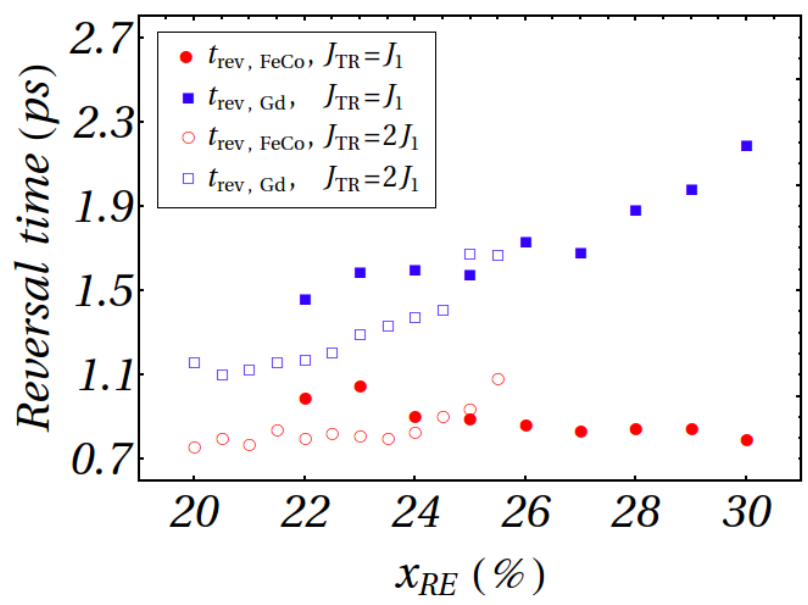

FIG. 5. (Color online) The reversal time of $\mathrm{FeCoGd}$ compound versus the rare-earth concentration, for different strength coupling strengths between both sublattices under the pulse fluence 40 $\mathrm{mJ} / \mathrm{cm}^{2}$.

in Refs. ${ }^{11,15}$ The dynamics is modelled by the Langevin dynamics based on Landau-Lifshitz-Gilbert equation of motion for localized atomic magnetic moments. The input electron temperature is dynamically varied using the two-temperature model, with parameters from Ref. ${ }^{11}$ First the system is equilibrated at $T=300 \mathrm{~K}$, after that the laser pulse with fluence $F=40 \mathrm{~mJ} / \mathrm{cm}^{2}$ is applied. The simulated system has the size $60^{3}$ magnetic moments with periodic boundary condition and we have performed averaging over several random configurations. In this case a more realistic coupling to the bath parameter $\lambda=0.02$ was used.

In Fig. 5 we present the reversal time of each sublattice (defined as time elapsed between the initial state and the instant of time at which the average magnetization starts to reverse its direction, i.e. crosses $m_{z, T M(R E)}=0$ point) versus the rare-earth concentration for two values of the inter-sublattice strength couplings. The Gd reversal time also corresponds to the total system reversal time. Note that apriori one should not expect direct correspondence between the relaxation and the switching time since these are two different quantities. In fact, the dynamics of the switching process is highly nonlinear and more factors such as a complex temperature profile play role in the behavior of this characteristic time scale. However, some features of the linear relaxation time is still seen in the behavior of this more complex quantity. We should mention that for $J_{T R}=2 J_{1}$ no switching is observed for large concentrations since the TM relaxation time is too slow. Also, in agreement with the predictions of the relaxation time which is larger for more concentrated systems and smaller for larger coupling strength (see Figs. 2 and 3), the Gd and Fe switching time also shows similar tendencies. However, the behavior of the TM is more complicated. For $J_{T R}=J_{1}$ (typical for $\mathrm{GdFeCo}$ coupling strength) it has a maximum and at high concentrations it decreases. These findings are in agree- ment with the fact that the switching window is larger for GdFeCo material with larger Gd concentration (although it will be finally suppressed), also reported in Ref. ${ }^{15}$ In recently published experimental results ${ }^{12}$ using the XMCD measurements in $\mathrm{GdFeCo}$ it was shown that the material is not homogeneous and the reversal starts in Gd-rich regions. The results presented in Fig. 5 (for $J_{T R}=J_{1}$ ) indicate that the reversal should start in region with the smallest $\mathrm{FeCo}$ reversal time. Those regions indeed would correspond to the largest Gd concentration and the minimum TM reversal time and the maximum field coming from the RE. For larger coupling strength $J_{T R}=2 J_{1}$, however, the TM reversal time as a function of $\mathrm{Gd}$ concentration monotonically increases. This indicates that in this case the reversal could start in regions with smaller $\mathrm{Gd}$ concentration.

\section{CONCLUSION}

To conclude, within the framework of the Landau-LifshitzBloch model for ferrimagnets we have analysed the temperature and concentration dependence of the relaxation rates of the "fast" and "slow" components of ferrimagnetic alloys. Our results indicate that the "fast" (TM) material experiences critical slowing down at the common Curie temperature, while the "slow" RE material does not do this. This effect is the reason of the reversal suppression for high pulse intensities. Both materials typically slow down as a function of the RE concentration although this dependence can be reverted for the TM for large inter-sublattice coupling strengths. This has a remarkable consequence on the switching time. We show that for the values of the coupling strength corresponding to typical parameters of the GdFeCo sublattice the reversal should start in Gd rich region. The situation may be opposite for compounds with stronger inter-sublattice couplings. Finally, we have presented here an analytical framework which allows a fast design and optimization of this class of materials. Our findings are important for their future applications in all-optical magnetic recording.

\section{ACKNOWLEDGMENTS}

This work was supported from CONICYT-FONDECYT Postdoctoral program fellowship under grant No. 3130678 (Chile); Spanish Ministry of Economy and Competitiveness under the grants FIS2010-20979-C02-02, MAT2013-47078C2-2-P and by the European Community's Seventh Framework Programme FP7/2007-2013 under grant agreement No. 281043, FEMTOSPIN; FONDECYT under the grant No. 1120764 and 1120356; Basal Program Center for Development of Nanoscience and Nanotechnology (CEDENNA); Millennium Scientific Initiative, P10-061-F, UTA-project No. 8750-12 (Chile) and Engineering and Physical Sciences Research Council Grant No. EP/L002922/1. 
1 A. Kirilyuk, A. V. Kimel and Th. Rasing, Rep. Prog.Phys. 76, 026501 (2013).

2 C. D. Stanciu, F. Hansteen, A. V. Kimel, A. Kirilyuk, A. Tsukamoto, A. Itoh, and Th. Rasing, Phys. Rev. Lett. 99, 047601 (2007).

3 I. Radu, K. Vahaplar, C. Stamm, T. Kachel, N. Pontius, H. A. Durr, T. A. Ostler, J. Barker, R. F. L. Evans, R. W. Chantrell, A. Tsukamoto, A. Itoh, A. Kirilyuk, Th. Rasing and A. V. Kimel, Nature 472, 205 (2011).

4 S. Alebrand, M. Gottwald, M. Hehn, D. Steil, M. Cinchetti, D. Lacour, E. E. Fullerton, M. Aeschlimann and S. Mangin, Appl. Phys. Lett.101, 162408 (2012).

5 A. R. Khorsand, M. Savoini, A. Kirilyuk, A. V. Kimel, A. Tsukamoto, A. Itoh, and Th. Rasing, Phys. Rev. Lett. 110, 107205 (2013).

6 S. Mangin, M. Gottwald, C-H. Lambert, D. Steil, V. Uhlir, L. Pang, M. Hehn, S. Alebrand, M. Cinchetti, G. Malinowski, Y. Fainman, M. Aeschlimann and E. E. Fullerton, Nat. Mater. 13, 286 (2014).

7 K. Vahaplar, A. M. Kalashnikova, A. V. Kimel, D. Hinzke, U. Nowak, R. Chantrell, A. Tsukamoto, A. Itoh, A. Kirilyuk, and Th. Rasing, Phys. Rev. Lett. 103, 117201 (2009).

8 T. A. Ostler, J. Barker, R. F. L. Evans, R. W. Chantrell, U. Atxitia, O. Chubykalo-Fesenko, S. El Moussaoui, L. Le Guyader, E. Mengotti, L. J. Heyderman, F. Nolting, A. Tsukamoto, A. Itoh, D. Afanasiev, B. A. Ivanov, A. M. Kalashnikova, K. Vahaplar, J. Mentink, A. Kirilyuk, Th. Rasing and A. V. Kimel, Nature Comm.
3, 666 (2012).

9 B. Koopmans, G. Malinowski, F. Dalla Longa, D. Steiauf, M. Fahnle, T. Roth, M. Cinchetti and M. Aeschlimann, Nature Mat. 9, 259265 (2010).

10 U. Atxitia and O. Chubykalo-Fesenko, Phys. Rev. B 84, 144414 (2011).

11 J. Barker, U. Atxitia, T. A. Ostler, O. Hovorka, O. ChubykaloFesenko, and R. W. Chantrell, Sci. Rep., 3, 3262 (2013).

12 C. E. Graves, A. H. Reid, T. Wang, B. Wu, S. de Jong, K. Vahaplar, I. Radu, D. P. Bernstein, M. Messerschmidt, L. Muller, R. Coffee, M. Bionta, S. W. Epp, R. Hartmann, N. Kimmel, G. Hauser, A. Hartmann, P. Holl, H. Gorke, J. H. Mentink, A. Tsukamoto, A. Fognini, J. J. Turner, W. F. Schlotter, D. Rolles, H. Soltau, L. Struder, Y. Acremann, A. V. Kimel, A. Kirilyuk, Th. Rasing, J. Stohr, A. O. Scherz and H. A. Durr, Nat. Mater. 12, 293 (2013).

13 U. Atxitia, P. Nieves, O. Chubykalo-Fesenko, Phys. Rev. B 86, 104414, (2012).

14 P. Nieves, U. Atxitia, R. W. Chantrell, and O. ChubykaloFesenko, J. Low Temp. Phys. 41, 949 (2015).

15 U. Atxitia, J. Barker, R.W. Chantrell and O. Chubykalo-Fesenko, Phys.Rev.B 89, 224421, (2014).

16 T. A. Ostler, R. F. L. Evans, R. W. Chantrell, U. Atxitia, O. Chubykalo-Fesenko, I. Radu, R. Abrudan, F. Radu, A. Tsukamoto, A. Itoh, A. Kirilyuk, T. Rasing, and A. Kimel, Phys. Rev. B 84, 024407 (2011).

17 U. Atxitia, T. Ostler, J. Barker, R. F. L. Evans, R. W. Chantrell, and O. Chubykalo-Fesenko, Phys. Rev. B, 87, 224417 (2013). 